\title{
Experimental investigation of photoluminescence dynamics of cavity polaritons under nonresonant excitation
}

\author{
Baoli Liu ${ }^{1}$, Zhongying $\mathrm{Xu}^{1}$, Bingshen Wang ${ }^{1}$, Yuanming Deng ${ }^{1}$, \\ Fuhua Yang ${ }^{1}$ and $\mathrm{W} \mathrm{K} \mathrm{Ge}^{2}$ \\ ${ }^{1}$ National Laboratory for Superlattices and Microstructures, Institute of Semiconductors, \\ Chinese Academy of Sciences, PO Box 912, Beijing 100083, People's Republic of China \\ 2 Department of Physics, Hong Kong University of Sciences and Technology, Konlow, \\ Hong Kong, People's Republic of China
}

Received 10 May 2001, in final form 4 July 2001

Published 30 August 2001

Online at stacks.iop.org/JPhysCM/13/8467

\begin{abstract}
Low-temperature time-resolved photoluminescence (PL) experiments have been performed on a semiconductor planar microcavity, which contains two sets of three $\operatorname{In}_{0.13} \mathrm{Ga}_{0.87} \mathrm{As} / \mathrm{GaAs}$ quantum wells embedded in a $3 \lambda / 2 \mathrm{GaAs}$ cavity. The spontaneous emission dynamics of both lower- and upper-branch polaritons is investigated as a function of exciton-cavity detuning under nonresonant optical excitation. It is found that the PL decay times of both branches are independent of cavity detuning while the PL rising kinetics of the lower- and upper-branch polaritons exhibits a significant difference. The rise time of the upper polarition branch shows a strong dependence on cavity detuning, while the rise time of the lower polarition branch is less sensitive to cavity detuning. Our results can be well understood in the framework of the theoretical prediction of Tassone et al.
\end{abstract}

\section{Introduction}

A strong coupling between the exciton and the cavity mode is one of the fundamental properties of semiconductor microcavities (MCs) [1-4]. It leads to the formation of new mixed excitonphoton modes, known as cavity polariton modes. Their properties strongly depend on the relative contents of exciton and photon: polaritons with a large photon fraction have a very steep dispersion curve and the density of states is considerably smaller than that of the bare excitons in quantum wells (QWs). In the following, we refer to lower polaritons (LPs) and upper polaritons (UPs) as mixed modes. This cavity effect is expected to modify the dynamics of spontaneous emission [5-7]. In particular, the energy relaxation in the polariton system will be strongly modified compared to that of the uncoupled excitons. On the other hand, 
due to the low reflectivity of the distributed Bragg reflectors (DBRs) at large angles in a real structure, there exist a lot of polariton modes which are not efficiently confined in the cavity and are known as the leaky modes of the DBRs [8]. In the past few years, there have been a number of theoretical and experimental investigations devoted to this interesting subject. Most of the work has concentrated on the dynamics in the strong-coupling region at $k / / \approx 0$, in which phonon scattering is significantly slowed down and the radiative recombination rates are sizably enhanced $[2,5]$. Experimentally, resonant optical excitation was used to study the spontaneous-emission dynamics of polaritons near $k_{/ /}=0$. The decay rate was found to be significantly modified with respect to the cavityless excitons and to depend very much on cavity detuning under the resonant optical excitation [6]. However, under nonresonant excitation the overall photoluminescence (PL) dynamics was found to be significantly different from that observed under resonant excitation. Tassone et al $[9,10]$ presented a theoretical investigation on the PL dynamics in strong-coupling semiconductor microcavities and found that the PL decay process is not much influenced by cavity detuning. The authors attributed their results to the existence of the leaky modes of DBRs, which are most populated among the radiative states. The authors also studied the dynamics of the LP and UP branch PL rise time and found that the LP branch PL rise time is detuning-independent while the UP branch PL dynamics shows a marked dependence on detuning. A mechanism involving different scattering processes was proposed to explain the above results. Although the theoretical predictions are very solid, little experimental work was found in the literature. Recently Müller et al [11] investigated polariton PL dynamics in their II-VI semiconductor microcavities under nonresonant excitation. The observed cavity detuning dependence of the PL decay time was attributed to the build-up of a bottleneck effect in the polariton relaxation processes.

In this work, we investigated the PL dynamics of an InGaAs/GaAs semiconductor microcavity under nonresonant excitation using time-resolved PL (TRPL) measurements. It is found that the measured decay times of the two polariton branches are almost the same and both independent of cavity detuning. However, the PL rise dynamics of the LP and UP branch are significantly different: the rise time of the LP branch is not sensitive to cavity detuning, while the rise time of the UP branch shows a strong dependence on cavity detuning. Our results can be well understood in the framework of the theoretical prediction of Tassone et al $[9,10]$.

\section{Experiments}

The semiconductor microcavity studied here was grown by molecular beam epitaxy (MBE) on a (100)-oriented GaAs substrate. The sample structure consists of 15 and 19 pairs of $\lambda / 4 \mathrm{GaAs} / \mathrm{AlAs}$ stacks as the top and bottom DBRs, respectively. Two groups of three $\mathrm{In}_{0.13} \mathrm{Ga}_{0.87} \mathrm{As} / \mathrm{GaAs}$ QWs with well width of $11 \mathrm{~nm}$ separated by a $15 \mathrm{~nm}$ GaAs barrier are placed at the antinodes of a $3 \lambda / 2 \mathrm{GaAs}$ cavity. The resonant wavelength of the planar microcavity under normal incidence was determined to be $900 \mathrm{~nm}$ by reflectivity measurement. The sample was fabricated with a slight wedge by keeping the sample unrotated during the growth of the cavity layer, so that the effective thickness of the cavity varied along the radial direction of the wafer. Therefore, variation of the exciton-cavity detuning can be obtained simply by moving the position of the excitation spot on the sample. The PL and TRPL measurements were performed at $T=12 \mathrm{~K}$. The maximum angle for collecting the luminescence signals is about $5^{\circ}$. In the experiment a $750 \mathrm{~nm}$ laser line was used as the excitation light source from a mode-locked Ti:sapphire femtosecond pulse laser at a repetition rate of $82 \mathrm{MHz}$ with $100 \mathrm{fs}$ pulse width. The laser spot diameter was about $150 \mu \mathrm{m}$ and the typical excitation power was $4 \mathrm{~mW}$. The signal from the sample was dispersed by a 


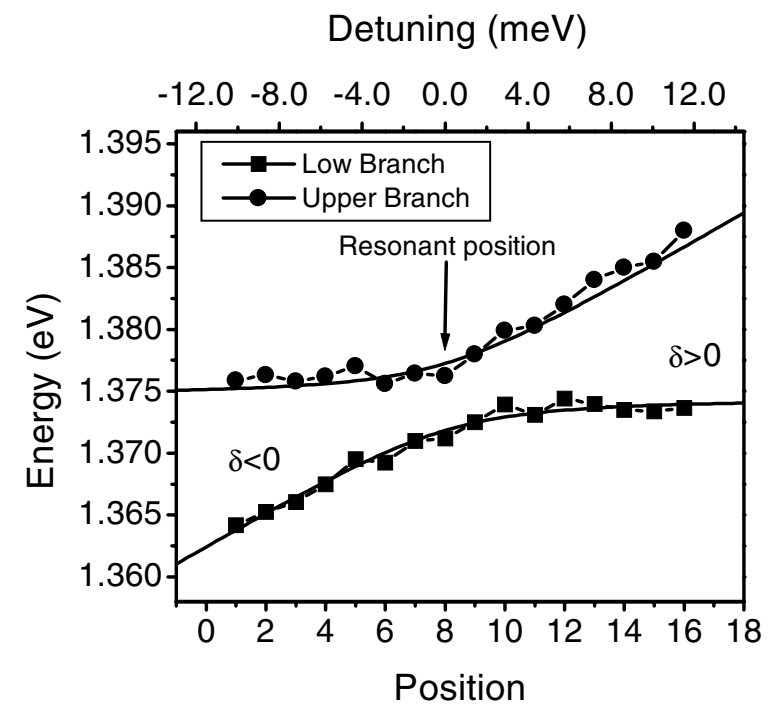

Figure 1. PL peak energy as a function of the position of the excitation spot on the sample (lower $x$-axis). The corresponding cavity-exciton detuning is indicated on the upper $x$-axis. The solid curves are calculated fittings and the arrow shows the resonant position.

Jobin-Yvon HR250 monochromator and detected by a photomultiplier tube in PL and by a 2D synchroscan streak camera in TRPL, which provides an overall temporal resolution less than 20 ps.

\section{Results and discussion}

The PL was measured at different positions of the sample. The variation of the position results in variation of the detuning between the exciton and cavity mode. As usual, we define detuning as $\delta=E_{\text {cav }}\left(k_{/ /}=0\right)-E_{\text {exc }}\left(k_{/ /}=0\right)$, where $E_{\text {cav }}\left(k_{/ /}=0\right)$ is the energy of the cavity mode and $E_{\text {exc }}\left(k_{/ /}=0\right)$ is the energy of the exciton. Figure 1 displays the measured PL peak energy as a function of the position of the excitation spot on the sample, which corresponds essentially to cavity-exciton detuning, as shown in the upper $x$-axis in the figure. From the figure, a well-behaved level anti-crossing constituting the LP and UP branches is observed, showing strong coupling between the exciton and cavity modes and indicating the high quality of the sample. The figure also gives an exciton energy of $\sim 1.375 \mathrm{eV}$ and the minimum exciton-photon mode splitting (Rabi splitting) of $\sim 5.4 \mathrm{meV}$.

We have also measured TRPL at the same position of the sample at normal incidence while measuring PL. Figures 2 and 3 present the measured PL decay curves of the LP and UP branches, respectively, at their respective peak energies for various positions. All curves exhibit a monoexponential decay with a time constant of $\sim 2300$ ps except for the PL decay from the UP branch at highest detunings, which will be discussed later. This indicates that the decay times of both branches are independent of the detunings.

The above result can be understood as follows: under a nonresonant excitation, the photogenerated carriers within the QWs rapidly cool down through the emission of LO phonons and form polaritons on the LP branch at large in-plane wave vectors through the emission of an acoustic phonon. Meanwhile, the upper branch polaritons at the electron-hole continuum energy are unlikely to be formed because of their small exciton content [10]. In 


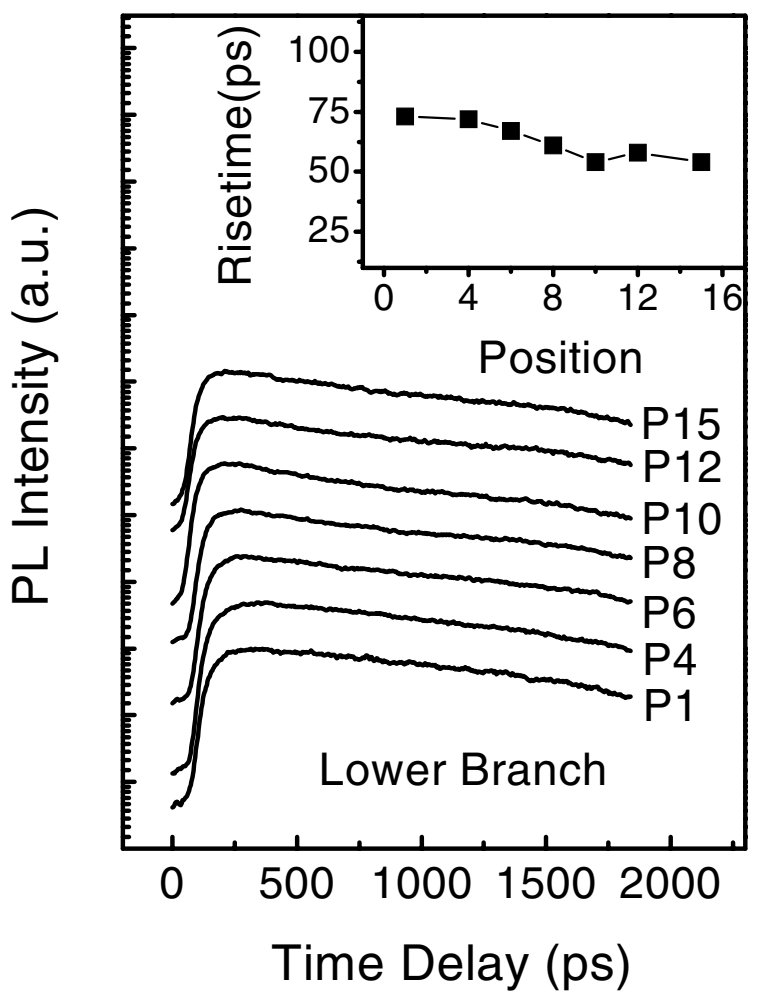

Figure 2. Time evolution of the PL intensity of the LP branch at the peak energy for various positions. Pi denotes the $i$ th detection position. The inset shows the rise times as a function of the position of the excitation spot on the sample.

figure 4, we plot the dispersion curves of the cavity polaritons for three different detunings as a function of the in-plane wave vector. Also shown are the dispersion curves for uncoupled exciton and cavity photons (dotted curves). It can be seen that the formed polaritons with large in-plane wave vectors mainly occupy region II of the LP branch. For a radiative recombination, the polariton states will further relax to the states at $k_{/ /} \approx 0$ through the emission of acoustic phonons and exciton-exciton scattering [12]. However, due to the existence of the bottleneck region (region I in the figure) and the lower final density of states [5, 13], most of the polariton states will radiatively recombine through the leaky modes of the LP branch. Moreover, the polariton states in the strong-coupling region $\left(k_{/ /} \approx 0\right)$ span only a small fraction of the total radiant modes (roughly 10\%) [2]. Most of the radiant polariton states lie in the leaky modes. Therefore, the total polariton population decay rate is solely determined by the dynamics within the lower (exciton-like) branch. In other words, the PL decay processes of the LP and UP branches are determined by the radiative recombination rate of the leaky modes. Consequently, the PL decay times of these two branch polaritons are approximately the same and independent of the detunings, as predicted by Tassone et al $[9,10]$.

It is noted from figure 3 that the decay of the PL from the UP branch is highly enhanced in the range of small delays at largest detunings. This is most likely due to the nonequilibrium distribution of the LP polaritons at large $k_{/ /}$. At short delay times, a large population of the LP branch is formed at the energy position close to the bottom energy of UP branch as predicted by Tassone et al in their theoretical calculation [9]. As the PL dynamics of the UP branch is 


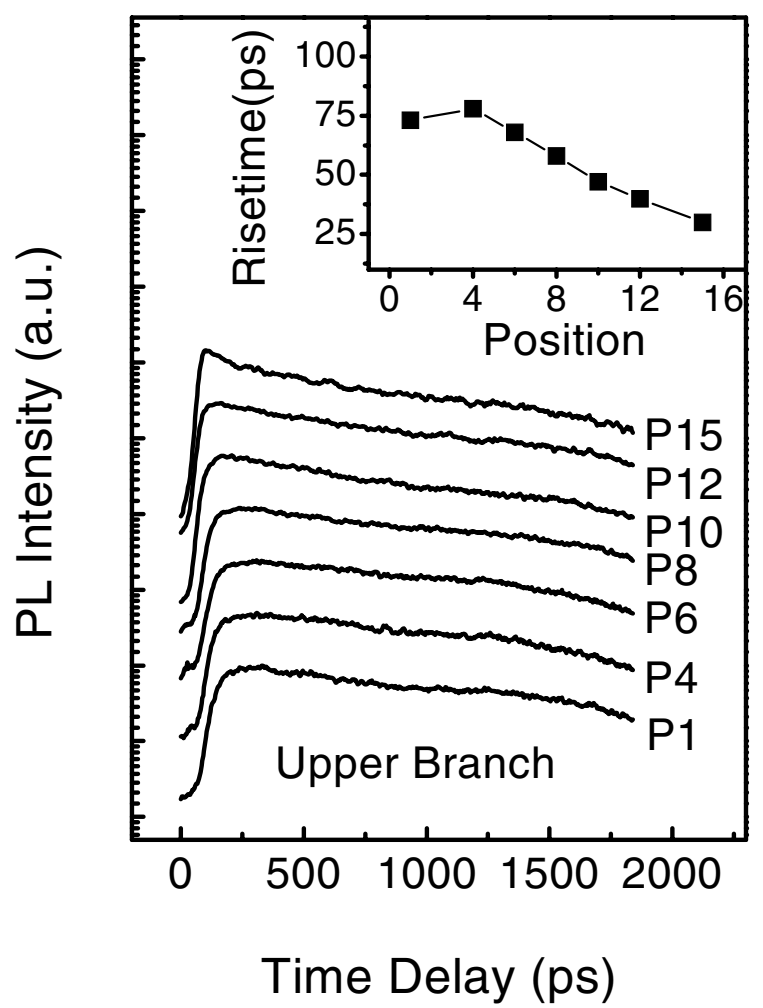

Figure 3. Time evolution of the PL intensity of the UP branch at the peak energy for various positions. $\mathrm{P} i$ denotes the $i$ th detection position. The inset shows the rise times as a function of the position of the excitation spot on the sample.

correlated with the polariton population and its relaxation rate in the LP branch with the same energy as the bottom of the UP branch, rapid decay is expected due to the fast relaxation of the nonequilibrium distribution. The details of this rapid decay process are beyond the scope of our discussion.

Now we discuss the PL rise processes of the LP and UP branches. In the insets of figures 2 and 3 the measured PL rise times are plotted as a function of the position of the excitation spot on the sample for the LP and UP branches, respectively. Here, the rise time is defined as the time required for the luminescence from 20 to $80 \%$ of the maximum intensity. It is found that the LP branch PL rise time is less sensitive to detuning, while the UP branch PL rise time shows a remarkable dependence on detuning: decreasing significantly with the increase of detuning from a negative to a large positive value. Our observation is in good agreement with the theoretical predictions of Tassone et al [10] and can be understood as follows.

It is known that the PL rise time corresponds to the population build-up time at $k_{/ /} \approx 0$. For the lower branch, the excitons cool down to the bottom of the exciton-like branch by phonon emissions. During further relaxation into the strong-coupling region, a bottleneck effect occurs. Therefore, the scattering rate from the bottleneck region to the strong-coupling region $\left(k_{/ /} \approx 0\right)$ is very small for the lower branch. In addition, the radiative recombination rate around $k_{/ /} \approx 0$ is much larger than the scattering rate into the bottom of the lower branch $[9,10]$. Therefore, the rise time of the PL at $k_{/ /}=0$ corresponds to the population build-up 

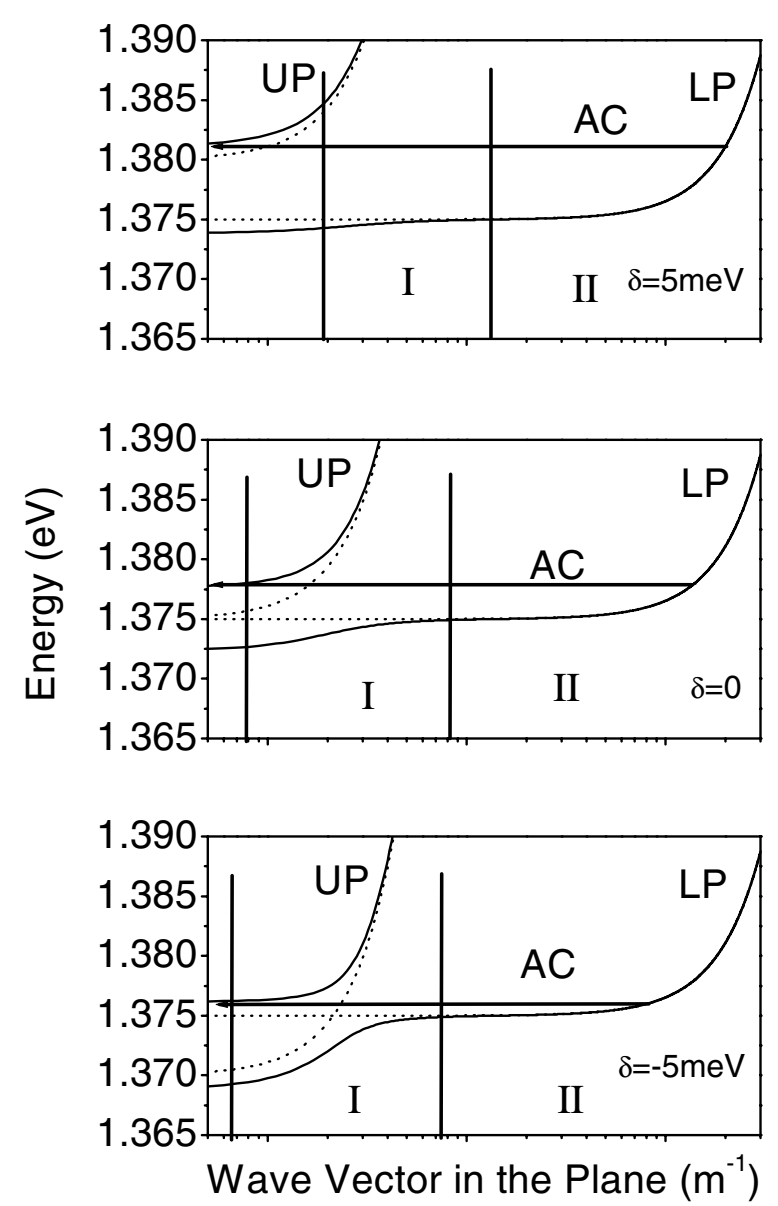

Figure 4. Dispersion curves of the upper and the lower cavity polariton modes as a function of the wave vector in the plane for three typical values of detuning. The dotted curves are the dispersion curves of the cavity without exciton and of the exciton without cavity. Regions I and II denote the region of the bottleneck and the region of the large- $k / /$ thermalized exciton.

time of the bottom of the exciton-like branch and is purely determined by this relaxation time. It is independent of detuning. On the other hand, we note from figure 4 that the curvature of the dispersion curves decreases when detuning increases from negative to positive values (see region I of figure 4). This results in the weakening of the bottleneck effect and an increase of acoustic phonon scattering rate from the weak-coupling region to the strong-coupling region $\left(k_{/ /} \approx 0\right)$. Therefore, the rise time of the LP branch shows some variation in detuning, as observed in our experiment (see the inset of figure 3 ).

For the UP branch, however, the mechanism for population is inter-branch scattering from excitons in the lower branch. We have already noted that the upper-branch polaritons at the electron-hole continuum energy are unlikely to be formed because of their small exciton content [10]. Excitons in a portion of the LP branch having the same energy as the bottom of the UP branch are involved in this inter-branch scattering process by acoustic-phonon emission or absorption, as denoted by $\mathrm{AC}$ in figure 4. This scattering rate is expected to be proportional to the modulus of the acoustic phonon wave vector [14]. When detuning varies from negative 
to positive the modulus of the phonon wave vector becomes larger and the scattering rate increases. As a result, the PL rise time of the UP branch becomes shorter. On the other hand, the population of the UP branch at $k_{/ /} \approx 0$ follows the population of the LP branch at the same energy. As the energy of the UP branch at $k_{/ /} \approx 0$ becomes higher for larger positive detunings, the cooling excitons reach these higher energies before reaching the lower energies for negative detunings. This effect also contributes to the detuning dependence of the PL rise time of the UP branch. It also explains the fact that the UP branch rise time is usually shorter than the LP branch one, as observed in our experiment. Finally we note that the measured rise times of the LP and UP branches are almost the same (77 versus 73 ps) at the largest negative detuning. This can be attributed to the fact that at larger negative detunings the UP branch becomes exciton-like (referring to figure 4); thus the PL rise time approaches the value found for the LP branch.

It is worth pointing out that when the bottom of the LP branch is lower than that of the UP branch at $k_{/ /} \approx 0$ for larger positive detunings, the UP branch cannot be populated through the emission or absorption of acoustic phonons due to the small acoustic phonon energy $\sim 1 \mathrm{meV}$. So the dynamics changes after the cooling of excitons below the bottom of the UP branch. In this case, the exciton-exciton scattering must be taken into account. The population of the UP branch at $k_{/ /} \approx 0$ can be produced through two dynamical paths [12]: (1) the initial states are two excitons in the nonradiative states and the final states are the upper polariton at $k_{/ /} \approx 0$ and another exciton in the nonradiative state, i.e. exciton+exciton $\rightarrow \mathrm{UP}+$ exciton; (2) the initial states are two excitons at opposite in-plane wave vectors $\left( \pm k_{/ /}\right)$and the final states are an upper polariton and a lower polariton at $k_{/ /} \approx 0$, i.e. exciton+exciton $\rightarrow \mathrm{UP}+\mathrm{LP}$. Therefore, we can observe experimentally the luminescence emitted from the UP branch.

\section{Conclusions}

We have investigated the PL dynamics of an InGaAs/GaAs semiconductor microcavity under nonresonant excitation using PL and TRPL measurements at $12 \mathrm{~K}$. It is found that the measured decay times of the two branch polaritons are almost the same and independent of cavity detuning. This result is in good agreement with the theoretical prediction of Tassone et al, indicating that the leaky modes of the DBRs dominate the whole recombination process. The PL rise dynamics of the lower- and upper-branch polaritons shows a significant difference: the rise time of the LP branch is less sensitive to cavity detuning, while the rise time of the UP branch presents a strong dependence on cavity detuning. A physical understanding based on the polariton relaxation and recombination process is given to explain our experimental results.

\section{Acknowledgments}

The work is supported by the National Science Foundation of China (Grants 19574047 and 19974045) and Grant HKUST 6135/97P from RGC, Hong Kong.

\section{References}

[1] Weisbuch C, Nishioka M, Ishikawa A and Arakawa Y 1992 Phys. Rev. Lett. 693314

[2] Bloch J and Marzin J Y 1997 Phys. Rev. B 562103

[3] Savona V, Andreani L C, Schwendimann P and Quattropani A 1995 Solid State Commun. 93733

[4] Dang Le Si, Heger D, Andre R, Boeuf F and Romestain R 1998 Phys. Rev. Lett. 813920

[5] Pau S, Björk G, Jacobson J, Cao H and Yamamoto Y 1995 Phys. Rev. B 517090 
[6] Sermage B, Long S, Abram I, Marzin J Y, Bloch J, Planel R and Thierry-Mieg V 1996 Phys. Rev. B 5316516

[7] Tanaka K, Nakamara T, Takamatsu W, Yamanishi M, Lee Y and Ishihara T 1995 Phys. Rev. Lett. 743380

[8] Savona V, Tassone F, Piermarocchi C, Quattropani A and Schwendimann P 1996 Phys. Rev. B 5313051

[9] Tassone F, Piermarocchi C, Savona V, Quattropani A and Schwendimann P 1996 Phys. Rev. B 53 R7642

[10] Tassone F, Piermarocchi C, Savona V, Quattropani A and Schwendimann P 1997 Phys. Rev. B 567554

[11] Müller M, Bleuse J, André R and Ulmer-Tuffigo H 1999 Physica B 272476

[12] Tassone F and Yamamoto Y 1999 Phys. Rev. B 5910830

[13] Emam-Ismail M, Astratov V N, Skolnick M S, Whittaker D M and Roberts J S 2000 Phys. Rev. B 621552

[14] Ridley B K 1988 Quantum Processes in Semiconductor 2nd edn (Oxford: Clarendon) p 130 